\title{
Applications of W Algebras to BF Theories, QCD, and 4D Gravity
}

\author{
R.P. Lano and V.G.J. Rodgers \\ Department of Physics and Astronomy \\ The University of Iowa \\ Iowa City, Iowa 52242-1479 \\ March 1992 \\ Dedicated to Ms. Miya Sonya Sioson \\ and to the memory of \\ Prof. Dwight Nicholson, Prof. Bob Smith, Prof. Chris Goertz, \\ Dr. Anne Cleary and Dr. Linhua Shan
}

\begin{abstract}
We are able to show that BF theories naturally emerge from the coadjoint orbits of $W_{2}$ and $w_{\infty}$ algebras which includes a Kac-Moody sector. Since QCD strings can be identified with a BF theory, we are able to show a relationship between the orbits and monopole-string solutions of QCD. Furthermore, we observe that when 4D gravitation is cast into a $\mathrm{BF}$ form through the use of Ashtekar variables, we are able to get order $\hbar$ contributions to gravity which can be associated with the $W_{2}$ anomaly. We comment on the relationship to gravitational monopoles.
\end{abstract}


Topological field theories are today considered an indispensable tool in the zeroth and first order attempts to understand many physical systems and mathematical issues. In particular BF and Chern-Simons theories have provided physicists with an enormous amount of insight into what we should look for in systems such as 3d quantum gravity [1], 4D gravity [2-3], superconductivity, and the London equations [4]. In this letter we would like to contribute to this wealth of knowledge by addressing the question of $\mathrm{BF}$ anomalies coming from the $w_{\infty}$ and $W_{2}$ algebras. We will relate these anomalies to QCD and 4D gravity. The methods we use are well known [5-10] and rely heavily on the existence of a symplectic two form for the infinite dimensional algebra. Therefore other $W_{n}$ algebras cannot be considered from this viewpoint. Our first calculations will be with respect to a three dimensional BF theory. Then we construct a four dimensional $\mathrm{BF}$ theory that enjoys $w_{\infty}$ symmetry. We will arrive at the BF theories "through the back door." In other words our approach is to ask, "given a specific symmetry, what action yields the associated Noether currents."

\section{Three Dimensional Systems}

Let us begin by studying a Kac-Moody algebra and the diffeomorphism of the circle parameter [11]. One recalls that there we have a Kac-Moody algebra associated with the group $\mathrm{G}$ given by

$$
\begin{aligned}
{\left[J_{N}^{\alpha}, J_{M}^{\beta}\right] } & =i f^{\alpha \beta \gamma} J_{N+M}^{\gamma}+N k \delta_{M+N, 0} \delta^{\alpha \beta} \\
{\left[L_{N}, J_{M}^{\alpha}\right] } & =-M J_{M+N}^{\alpha} \\
{\left[L_{N}, L_{M}\right] } & =(N-M) L_{N+M}+\frac{c}{12}\left(N^{3}-N\right) \delta_{N+M, 0}
\end{aligned}
$$

where $c=\frac{2 k \operatorname{Dim}(G)}{2 k+c_{v}}, \operatorname{Dim}(G)$ is the dimension of the group and $c_{v}$ is the value of the quadratic Casimir in the adjoint representation. Now the choice of representations depends on the topology of the manifold. Let us choose the representation,

$$
\begin{aligned}
L_{N} & =i \exp ^{i N \theta} \partial_{\theta} \\
J_{N}^{\alpha} & =\tau^{\alpha} \exp ^{i N \theta} .
\end{aligned}
$$


We normalize the generators so that $\operatorname{Tr}\left(\tau^{\alpha} \tau^{\beta}\right)=\delta^{\alpha \beta}$. Let us denote $\left(L_{A}, J_{B}^{\beta}, \rho\right)$ as a centrally extended adjoint vector. Then from the commutation relations above one may write the adjoint action on the adjoint vectors as

$$
\begin{aligned}
& \left(L_{A}, J_{B}^{\beta}, \rho\right) *\left(L_{N^{\prime}}, J_{M^{\prime}}^{\alpha^{\prime}}, \mu\right)= \\
& \left(\left(A-N^{\prime}\right) L_{A+N^{\prime}},\right. \\
& -M^{\prime} J_{A+M^{\prime}}^{\alpha^{\prime}}+B J_{B+N^{\prime}}^{\beta}+i f^{\beta \alpha^{\prime} \lambda} J_{B+M^{\prime}}^{\lambda}, \\
& \left.\frac{c}{12}\left(A^{3}-A\right) \delta_{A+N^{\prime}, 0}+B k \delta^{\alpha^{\prime} \beta} \delta_{B+M^{\prime}, 0}\right) .
\end{aligned}
$$

Now we need the coadjoint representation and a suitable inner product. Let $\left(\tilde{L}_{N}, \tilde{J}_{M}^{\alpha}, \tilde{\mu}\right)$ denote an element of the coadjoint representation. A suitable pairing is simple

$$
\left\langle\left(\tilde{L}_{N}, \tilde{J}_{M}^{\alpha}, \tilde{\mu}\right) \mid\left(L_{N^{\prime}}, J_{M^{\prime}}^{\alpha^{\prime}}, \mu^{\prime}\right)\right\rangle=\delta^{N, N^{\prime}}+\delta^{\alpha, \alpha^{\prime}} \delta_{M, M^{\prime}}+\mu \tilde{\mu}
$$

With this the adjoint action on the coadjoint representation can be extracted by requiring that

$$
\begin{array}{r}
\left\langle\left(\tilde{L}_{N}, \tilde{J}_{M}^{\alpha}, \tilde{\mu}\right) \mid\left(L_{A}, J_{B}^{\beta}, \rho\right) *\left(L_{N^{\prime}}, J_{M^{\prime}}^{\alpha^{\prime}}, \mu\right)\right\rangle= \\
-\left\langle\left(L_{A}, J_{B}^{\beta}, \rho\right) *\left(\tilde{L}_{N}, \tilde{J}_{M}^{\alpha}, \tilde{\mu}\right) \mid\left(L_{N^{\prime}}, J_{M^{\prime}}^{\alpha^{\prime}}, \mu\right)\right\rangle .
\end{array}
$$

This means that the action of an adjoint vector on a coadjoint vector yields a new coadjoint vector through,

$$
\begin{aligned}
& \left(L_{A}, J_{B}^{\beta}, \rho\right) *\left(\tilde{L}_{N}, \tilde{J}_{M}^{\alpha}, \tilde{\mu}\right)= \\
& \left(-(2 A-N) \tilde{L}_{N-A}-B \delta^{\alpha \beta} \tilde{L}_{M-B}-\frac{\tilde{\mu} c}{12}\left(A^{3}-A\right) \tilde{L}_{-A},\right. \\
& \left.(M-A) \tilde{J}_{M-A}^{\alpha}-i f^{\beta \nu \alpha} \tilde{J}_{M-B}^{\nu}-\tilde{\mu} B k \tilde{J}_{-B}^{\beta}, 0\right) .
\end{aligned}
$$

By setting this equation to zero one can determine the isotropy algebra for the covector $\left(\tilde{L}_{N}, \tilde{J}_{M}^{\alpha}, \tilde{\mu}\right)$. Instead of using components, let us write $F=(f(\theta), \hat{h}(\theta), a)$ as an arbitrary adjoint vector and $B=(b(\theta), h(\theta), \mu)$ as an arbitrary coadjoint vector, where $f, \hat{h}, b$, and $h$ are functions constructed from the above mentioned basis vectors. Then Eq. (1) may be written as

$$
\begin{aligned}
& \delta_{F} B \equiv(f(\theta), \hat{h}(\theta), a) *(b(\theta), h(\theta), \mu)= \\
& -\left(2 f^{\prime} b+b^{\prime} f+i \frac{c \mu}{12} f^{\prime \prime \prime}+\operatorname{Tr}\left[h \hat{h}^{\prime}\right], h^{\prime} f+h f^{\prime}+[\hat{h} h-h \hat{h}]+i k \mu \hat{h}^{\prime}, 0\right),
\end{aligned}
$$


where' denotes $\partial_{\theta}$. Those adjoint vectors, $F$, that leave $B$ invariant will generate the isotropy group for $B$. The group action of $\mathrm{G}$ on $B$ is generated by the adjoint representation for those elements of the group that are connected to the identity. Equation (1a) then determines the tangent space on the orbit of $B$. Thus for coadjoint elements $B_{1}$ and $B_{2}$, we may construct the usual symplectic two form by writing

$$
\Omega_{B}\left(B_{1}, B_{2}\right)=\left\langle B \mid\left[F_{1}, F_{2}\right]\right\rangle
$$

where for example $\delta_{F_{1}} B=B_{1}$.

Next we will need the two cocycle associated with the central extension for our representation. For adjoint elements $(L, J, \mu)$ and $(\hat{L}, \hat{J}, \hat{\mu})$ we may define the two cocycle as,

$$
\begin{aligned}
& C_{\mathrm{DiffS}^{1} \times \mathrm{SU}(\mathrm{N})}((L, J, \mu),(\hat{L}, \hat{J}, \hat{\mu}))= \\
& \frac{i c}{48 \pi} \int_{0}^{2 \pi}\left(L(\theta) \hat{L}(\theta)^{\prime \prime \prime}-\hat{L}(\theta) L(\theta)^{\prime \prime \prime}\right) d \theta \\
& +\frac{k}{2 \pi} \int_{0}^{2 \pi} \operatorname{Tr} J(\theta) \partial_{\theta} \hat{J}(\theta) d \theta .
\end{aligned}
$$

Now the idea of constructing actions from coadjoint orbits is very simple. One simply considers the orbit of $B$ as a manifold. Since $G$ action on $B$ corresponds to a new point on the orbit, we may think of $\mathrm{G}$ modulo elements of the isotropy group of $B$ as parameterizing the orbit. Then consider a two parameter family of group elements living on the orbit. These two parameters can be associated with a dynamical variable such as time, $\tau$ and a one parameter $(\lambda)$ family of maps from the identity (say) to a fixed element $\tilde{g}(\lambda=1, \tau)$. Thus our group elements may be written as $\tilde{g}(\lambda, \tau)$ and these correspond to generic Kac-Moody group and diffeomorphism group elements. We need to know the tangent covectors associated with infinitesimal transformations along the flow of $\lambda$ and $\tau$. Then one may simply write that

$$
S_{B}=\int d \lambda d \tau \Omega\left(B_{\lambda}^{\tilde{g}(\lambda, \tau)}, B_{\tau}^{\tilde{g}(\lambda, \tau)}\right)=\int d \lambda d \tau\left\langle B_{\tilde{g}(\lambda, \tau)} \mid\left[F_{\lambda}^{\tilde{g}(\lambda, \tau)}, F_{\tau}^{\tilde{g}(\lambda, \tau)}\right]\right\rangle .
$$

Here $F_{\lambda}^{\tilde{g}(\lambda, \tau)}$ and $F_{\tau}^{\tilde{g}(\lambda, \tau)}$ are the directional derivatives (adjoint vectors) associated with flow along $\lambda$ and $\tau$ respectively at the point determined by $\tilde{g}(\lambda, \tau)$. We simply need to disentangle this expression for our purposes. Because of the central extensions, the 
coadjoint action of the group will in general have an inhomogeneous term. One can show that [9] in these cases the action is

$$
S=\int d \lambda d \tau\left(\left\langle b \mid\left[\tilde{g}^{-1} F_{\lambda}^{\tilde{g}} \tilde{g}, \tilde{g}^{-1} F_{\tau}^{\tilde{g}} g\right]\right\rangle+\mu c\left(\tilde{g}^{-1} F_{\lambda}^{\tilde{g}} \tilde{g}, \tilde{g}^{-1} F_{\tau}^{\tilde{g}} \tilde{g}\right)\right)
$$

where the centrally extended coadjoint vector in consideration here is $B=(b, \mu)$, with $b$ representing all classical components and $\mu$ representing the central extensions. In the above $\tilde{g}^{-1} F_{\tau}^{\tilde{g}} \tilde{g}$ represents the pull back of the adjoint vector to the identity and $b$ is evaluated at the identity. With this we find that

$$
\begin{aligned}
& F_{\lambda}^{\tilde{g}}=\left(\partial_{\lambda} s(\theta, \lambda, \tau), \partial_{\lambda} g g^{-1}, 0\right) \\
& F_{\tau}^{\tilde{g}}=\left(\partial_{\tau} s(\theta, \lambda, \tau), \partial_{\tau} g g^{-1}, 0\right)
\end{aligned}
$$

and

$$
\begin{aligned}
& \tilde{g}^{-1} F_{\lambda}^{\tilde{g}} \tilde{g}=\left(\frac{\partial_{\lambda} s(\theta, \lambda, \tau)}{\partial_{\theta} s}, g^{-1} \partial_{\lambda} g, 0\right) \\
& \tilde{g}^{-1} F_{\tau}^{\tilde{g}} \tilde{g}=\left(\frac{\partial_{\tau} s(\theta, \lambda, \tau)}{\partial_{\theta} s}, g^{-1} \partial_{\tau} g, 0\right)
\end{aligned}
$$

where $s(\theta, \lambda, \tau)$ corresponds to a two parameter family of diffeomorphisms of the circle parameter and $g(\theta, \lambda, \tau)$ is a two parameter family of Kac-Moody group elements. With all of this in place we arrive at our result which corresponds to an action that is both Kac-Moody and diffeomorphism invariant.

$$
\begin{aligned}
& S=\int b(\theta)\left[\frac{\partial_{\lambda} s}{\partial_{\theta} s} \frac{\partial}{\partial \theta}\left(\frac{\partial_{\tau} s}{\partial_{\theta} s}\right)-\frac{\partial_{\tau} s}{\partial_{\theta} s} \frac{\partial}{\partial \theta}\left(\frac{\partial_{\lambda} s}{\partial_{\theta} s}\right)\right] d \lambda d \tau d \theta \\
& +\frac{k}{2 \pi} \int \operatorname{Tr}\left[h(\theta)\left\{\frac{\partial_{\lambda} s}{\partial_{\theta} s} \frac{\partial}{\partial \theta}\left(g^{-1} \partial_{\tau} g\right)-\frac{\partial_{\tau} s}{\partial_{\theta} s} \frac{\partial}{\partial \theta}\left(g^{-1} \partial_{\lambda} g\right)+\left[g^{-1} \partial_{\lambda} g, g^{-1} \partial_{\tau} g\right]\right\}\right] d \lambda d \tau d \theta \\
& +\frac{c\left(k, c_{v}, 26\right) \mu}{48 \pi} \int\left[\frac{\partial_{\theta}^{2} s}{\left(\partial_{\theta} s\right)^{2}} \partial_{\tau} \partial_{\theta} s-\frac{\left(\partial_{\theta}^{2} s\right)^{2}\left(\partial_{\tau} s\right)}{\left(\partial_{\theta} s\right)^{3}}\right] d \theta d \tau-\frac{n \mu}{4 \pi} \int \operatorname{Trg}^{-1} \frac{\partial g}{\partial \theta} g^{-1} \frac{\partial g}{\partial \tau} d \theta d \tau \\
& +\frac{k \mu}{4 \pi} \int \operatorname{Trg}^{-1} \frac{\partial g}{\partial \theta}\left[g^{-1} \frac{\partial g}{\partial \lambda}, g^{-1} \frac{\partial g}{\partial \tau}\right] d \theta d \tau d \lambda .
\end{aligned}
$$

In the above action we have written $c\left(k, c_{v}, 26\right)$ as the central extension shifted by 26 in accord with reparameterization ghosts that arise from a covariant treatment. The covector defining the orbit is $B=(b(\theta), h(\theta), \mu)$. By setting Eq. (1a) to zero one can 
recover the isotropy group for $B$. Keep in mind that $b(\theta)$ is a quadratic differential and the $h(\theta)$ is valued in the Lie algebra of $\mathrm{G}$.

Now the fact that this is a BF theory in three dimensions can be seen from the first two lines of Eq. (4). Indeed by writing the Kac-Moody $\times$ Diff $S^{1}$ valued curvature two forms as

$$
\begin{aligned}
\mathbf{F}_{\lambda \tau} & =\frac{\partial_{\lambda} s}{\partial_{\theta} s} \frac{\partial}{\partial \theta}\left(g^{-1} \partial_{\tau} g\right)-\frac{\partial_{\tau} s}{\partial_{\theta} s} \frac{\partial}{\partial \theta}\left(g^{-1} \partial_{\lambda} g\right)+\left[g^{-1} \partial_{\lambda} g, g^{-1} \partial_{\tau} g\right] \\
& +\left(\frac{\partial_{\lambda} s}{\partial_{\theta} s} \frac{\partial}{\partial \theta}\left(\frac{\partial_{\tau} s}{\partial_{\theta} s}\right)-\frac{\partial_{\tau} s}{\partial_{\theta} s} \frac{\partial}{\partial \theta}\left(\frac{\partial_{\lambda} s}{\partial_{\theta} s}\right)\right)
\end{aligned}
$$

and writing the corresponding one form $\mathbf{B}$ as $\mathbf{B}_{\theta}=h(\theta)+b(\theta)$, and all other terms zero, we see that the above action is just

$$
S=\int \operatorname{Tr}\left(\epsilon^{i j k} B_{i} F_{j k}\right) d \lambda d \tau d \theta=\int \operatorname{Tr} B_{\wedge} F .
$$

Note that the last term of $\mathbf{F}$ and the $b(\theta)$ term of $\mathbf{B}$ are related to an abelian subalgebra. Let us remark that throughout we have assumed a three dimensional topology of $S^{1} \times$ $R^{2}$. If one wishes to change the topology, this will modify the central extension term. However the analysis would be straightforward. Now the last two summands of Eq. (4) are order $\hbar$ contributions to the theory. Since a BF theory in three dimensions may be written as a Chern-Simons theory $[1,12]$ we find that a WZNW model and a LiouvillePolyakov model appear at the first level of quantum corrections. In fact these terms may be viewed as the effective action of a BF theory or the bosonization of a fermionic theory coupled to an external gauge potential and 2D gravity. Let us discuss some of the applications of this model.

In Ref. [13] the author has argued that the vacuum sector for QCD may best be described as a WZNW model immersed into a four dimensional manifold. By considering an $\mathrm{SU}(3)$ Kac-Moody group we can recover that string theory modulo self-intersection terms. We observe that the gravity from the string world surface introduces an anomaly via the Liouville-Polyakov term. This term will break the scale invariance and may possibly be related to $\Lambda_{Q C D}$. It is plausible that since the two dimensional gravity associated with the string world surface arises from the induced metric of four dimensions that the scale anomaly of QCD is due to gravity. This would imply that the dilaton arises from 
quantum gravity effects. Also the magnetic-instanton sector [14] of QCD in the stringlike phase is directly related to the classical BF part of our action. By choosing the coadjoint vector $\mathbf{B}=(b, h, \mu)$ where $h$ and $b$ are $S^{1}$ invariant field configurations (by this we mean that they are $\theta$ independent), and from Eq. (1a),

$$
\begin{aligned}
& \quad \delta_{F} B \equiv(f(\theta), \hat{h}(\theta), a) *(b(\theta), h(\theta), \mu)= \\
& -\left(2 f^{\prime} b+b^{\prime} f+i \frac{c \mu}{12} f^{\prime \prime \prime}+\operatorname{Tr}\left[h \hat{h}^{\prime}\right], h^{\prime} f+h f^{\prime}+[\hat{h} h-h \hat{h}]+i k \mu \hat{h}^{\prime}, 0\right),
\end{aligned}
$$

one sees that the field space is equivalent to $\mathrm{G} / \mathrm{H} \otimes \Omega(\mathrm{G}) \otimes \frac{\operatorname{Diff} S^{1}}{S^{1}}$, where $\mathrm{H}$ is the isotropy group of $g$ and $\Omega(G)$ is the loop group of $\mathrm{G}$. The division by $S^{1}$ is automatic in this case since Eq. (1a) is still divisible by rigid rotations from the Diff $\mathrm{S}^{1}$ sector. Note that an $S L(2, C)$ invariant configuration $b(\theta)$ will spoil the isotropy of $h$. Different embeddings of $\mathrm{H}$ in $\mathrm{G}$ will have to be related to inequivalent self-dual monopoles. As a final note, the value of $c\left(k, c_{v}, 26\right)=26-\frac{8 \times 2 k}{2 k+6}$ (for our normalization of the generators $c_{v}=6$ as opposed to 3 for the standard normalization $\left.\operatorname{Tr}\left(\tau^{a} \tau^{b}\right)=\frac{1}{2} \delta^{a b}\right)$. We may also set the value of $k=1$ to yield $c\left(k, c_{v}, 26\right)=24$.

The action also describes a generic $\mathrm{G} / \mathrm{H}$ coset model [15] coupled to the Liouville mode. Observe that in the absence of the Liouville mode ( $\operatorname{set} s(\theta, \lambda, \tau)=0$ ) one may integrate the $h(\theta)\left[g^{-1} \partial_{\lambda} g, g^{-1} \partial_{\tau} g\right]$ term by parts [7] to get $h(\theta) g^{-1} \partial_{\tau} g$. This is just the light-cone gauge fixed contribution of the G-O coset models to a WZNW theory.

Since three dimensional gravity is believed to be a BF theory [1] the action of Eq. (4) has a further application. By considering an $\operatorname{ISO}(2,1)$ gauge group and a three manifold with $R^{2} \times S^{1}$ topology, the action above corresponds to a light-cone gauge fixed $\mathrm{BF}$ theory describing a $3 \mathrm{D}$ gravitational theory and its order $\hbar$ corrections. The Eq. (5a) and Eq. (5b) correspond to the curvature contributions from the frame fields (as functions of $s(\theta)$ and its derivatives) and the spin connection $\left(g^{-1} \partial g\right)$. The WZNW and Liouville-Polyakov terms serve as the quantum corrections to this particular 3D topology. One may consider an $R^{3}$ topology by using a different representation for the Virasoro and Kac-Moody generators such as $L_{N}=z^{1-N} \partial_{z}$ and $J_{M}^{\alpha}=\tau^{\alpha} z^{-M}$. In any case the particular choice of the field $\mathbf{B}=(b(\theta), h(\theta), \mu)$ will choose the coadjoint orbit. Gravitational monopoles may be related to certain isotropy groups of $\mathbf{B}$ just as in the QCD case. 


\section{Four Dimensional Systems}

Recently a great deal of research has gone into identifying infinite dimensional symmetries in self-dual four dimensional gravity [16-19]. In fact $w_{\infty}$ (for a review see Ref. [20]) has been identified as a symmetry in self dual four dimensional systems through symplectic diffeomorphisms (Diff $M^{2}$ ) on two manifolds (i.e., they correspond to coordinate transformations of the two dimensional system which have unit Jacobian). At the same time, the work of Ashtekar et al. [21] has suggested that a self-dual formulation may be a more natural framework in which to study quantum gravity in four dimensions. We would like to use the underlying symplectic geometry of $w_{\infty}$ along with a contraction of an SU(N) Kac-Moody algebra in order to probe self-dual Yang-Mills systems with these symmetries. Our hope is to provide some insight into the quantum corrections to these theories.

Let us begin by writing a suitable representation for $w_{\infty}$ and the $\mathrm{SU}(\mathrm{N})$ sectors. The commutations relations are given by

$$
\begin{aligned}
{\left[W_{M}^{i}, W_{N}^{j}\right] } & =((j+1) M-(i+1) N) W_{M+N}^{i+j}+\frac{c}{12}\left(M^{3}-M\right) \delta_{M+N, 0} \delta^{i, 0} \delta^{j, 0} \\
{\left[J_{M}^{i}(\alpha), J_{N}^{j}(\beta)\right] } & =i f^{\alpha \beta \gamma} J_{M+N}^{i+j}(\gamma)+M k \delta_{M+N, 0} \delta^{\alpha \beta} \delta^{i, 0} \delta^{j, 0} \\
{\left[W_{N}^{i}, J_{M}^{j}(\alpha)\right] } & =(N j-M i-M) J_{M+N}^{i+j}(\alpha),
\end{aligned}
$$

where the central extension $c$ is related to the Kac-Moody central extension by $c=$ $\frac{2 k \operatorname{DimG}}{2 k+c_{v}}$. For the generators $W_{M}^{i}$ and $J_{M}^{i}(\alpha), i \geq 0, M$ is any integer and $\alpha=1, \ldots N^{2}-1$. Explicitly the classical part of the generators may be written as

$$
\begin{aligned}
W_{N}^{i} & =\partial_{x}\left(x^{N+1+i} y^{i+1}\right) \partial_{y}-\partial_{y}\left(x^{N+1+i} y^{i+1}\right) \partial_{x} \\
J_{N}^{i}(\alpha) & =\tau^{\alpha} x^{N+i} y^{i}
\end{aligned}
$$

with $\tau^{\alpha}$ representing the generators of SU(N). The function $x^{N+1+i} y^{i+1}$ will be considered the "Hamiltonian" for the vector field $W_{N}^{i}$. Note that it is sufficient to compute the Poisson bracket of the Hamiltonians to get the algebra for the Diff $R^{2}$ sector. The use of Poisson brackets will facilitate our computations in a few moments. With this representation we will promote our coordinates $x$ and $y$ to complex coordinates to facilitate the construction of the two cocycle. In other words we will simply use $\oint d z \frac{z^{p-1}}{2 \pi}=\delta_{p, 0}$. 
Let $f$ and $g$ be two arbitrary generators in the $w_{\infty}$ sector. Then we may write the two cocycle as

$$
C_{W_{\infty}}(f, g)=\frac{-c}{48 \pi^{2}} \oint d x d y \frac{\partial_{x} \partial_{y} f \partial_{x}^{2} \partial_{y} g}{y}
$$

Similarly for $\rho$ and $\gamma$ as arbitrary generators of the $\mathrm{SU}(\mathrm{N})$ sector we have

$$
C_{S U(N)}(\rho, \gamma)=\frac{-k}{4 \pi^{2}} \oint d x d y \frac{\partial_{x} \rho \gamma}{y}
$$

For the coadjoint vectors we will use the notation $\tilde{W}_{M}^{i}$ and $\tilde{J}_{M}^{i}(\alpha)$. A pairing between the adjoint vectors and coadjoint vectors in the $w_{\infty}$ sector is

$$
\left\langle\tilde{W}_{M}^{i} \mid W_{N}^{j}\right\rangle=\frac{-1}{4 \pi^{2}} \oint d x d y \tilde{W}_{M}^{i} W_{N}^{j}=\delta^{i, j} \delta_{M, N}
$$

so explicitly we find that $x^{-(M+i+2)} y^{-(i+2)}$ is the dual Hamiltonian for $\tilde{W}_{M}^{i}$ and for the Kac-Moody sector,

$$
\left\langle\tilde{J}_{M}^{i}(\alpha) \mid J_{N}^{j}(\beta)\right\rangle=\frac{-1}{4 \pi^{2}} \oint d x d y \operatorname{Tr}\left(\tilde{J}_{M}^{i}(\alpha) J_{N}^{j}(\beta)\right)=\delta^{i, j} \delta_{M, N} \delta^{\alpha \beta},
$$

so that our coadjoint vectors are $\tilde{J}_{N}^{j}(\alpha)=\tau^{\alpha} x^{-(N+j+1)} y^{-(j+1)}$ where $j \geq 0$.

Now we need to compute the coadjoint action of the group. Let $(a, \rho, t)$ be an element of the adjoint representation where the $a$ is the "Hamiltonian" of a symplectic diffeomorphism, $\rho$ is valued in the $\mathrm{SU}(\mathrm{N})$ sector, and $t$ is a central extension. Using this notation we may write the action of an adjoint vector on another adjoint vector as

$$
(a, \rho, t) *\left(a^{\prime}, \rho^{\prime}, t^{\prime}\right)=\left(\left\{a, a^{\prime}\right\},\left\{a, \rho^{\prime}\right\}+\left\{\rho, a^{\prime}\right\}+\left[\rho, \rho^{\prime}\right], C_{W_{\infty}}\left(a, a^{\prime}\right)+C_{S U(N)}\left(\rho, \rho^{\prime}\right)\right)
$$

In order to get the adjoint action on a coadjoint vector we consider the coadjoint vector $(\tilde{b}, \tilde{\gamma}, \tilde{t})$ paired with the adjoint element $\left(a^{\prime}, \rho^{\prime}, t^{\prime}\right)$, i.e., $\left\langle(\tilde{b}, \tilde{\gamma}, \tilde{t}) \mid\left(a^{\prime}, \rho^{\prime}, t^{\prime}\right)\right\rangle$. Then by Leibnitz rule we have that

$$
\left\langle(a, \rho, t) *(\tilde{b}, \tilde{\gamma}, \tilde{t}) \mid\left(a^{\prime}, \rho^{\prime}, t^{\prime}\right)\right\rangle+\left\langle(\tilde{b}, \tilde{\gamma}, \tilde{t}) \mid(a, \rho, t) *\left(a^{\prime}, \rho^{\prime}, t^{\prime}\right)\right\rangle=0
$$

From Eq. (7) this implies that

$$
(a, \rho, t) *(\tilde{b}, \tilde{\gamma}, \tilde{t})=\left(\{a, \tilde{b}\}+\{\rho, \tilde{\gamma}\}-\frac{c \tilde{t}}{12} \frac{\partial_{x}^{3} \partial_{y} a}{y^{2}},\{a, \tilde{\gamma}\}+[\rho, \tilde{\gamma}]-k \tilde{t} \frac{\partial_{x} \rho}{y}, 0\right)
$$


where we have used the Poisson bracket for all W,W and W,J commutators.

With all of this in place we may now write the action for the coadjoint vector $\mathbf{B}=(b(x, y), \gamma(x, y), \tilde{t})$ as,

$$
\begin{aligned}
S= & \frac{-1}{4 \pi^{2}} \int b(x, y)\left\{H_{\lambda}, H_{\tau}\right\} d^{4} x \\
& \frac{-1}{4 \pi^{2}} \int \operatorname{Tr} \gamma(x, y)\left(\left\{H_{\lambda}, g^{-1} \partial_{\tau} g\right\}-\left\{H_{\tau}, g^{-1} \partial_{\lambda} g\right\}+\left[g^{-1} \partial_{\lambda} g, g^{-1} \partial_{\tau} g\right]\right) d^{4} x \\
& \frac{-c \tilde{t}}{48 \pi^{2}} \int \frac{1}{y}\left(\partial_{x} \partial_{y} H_{\lambda}\right) \partial_{x}^{2} \partial_{y} H_{\tau} d^{4} x \\
& \frac{-k \tilde{t}}{4 \pi^{2}} \int \frac{1}{y} \partial_{x}\left(g^{-1} \partial_{\lambda} g\right)\left(g^{-1} \partial_{\tau} g\right) d^{4} x,
\end{aligned}
$$

where the adjoint vectors responsible for transport along the $\lambda$ and $\tau$ directions is given by

$$
\begin{aligned}
& \tilde{g}^{-1} F_{\lambda}^{\tilde{g}} \tilde{g}=\left(H_{\lambda}, g^{-1} \partial_{\lambda} g, 0\right) \\
& \tilde{g}^{-1} F_{\tau}^{\tilde{g}} \tilde{g}=\left(H_{\tau}, g^{-1} \partial_{\tau} g, 0\right) .
\end{aligned}
$$

The Hamiltonians $H_{\lambda}$ and $H_{\tau}$ are related to a symplectic diffeomorphism of the coordinates $x$ and $y$ by $\partial_{\rho} H_{\lambda}=\frac{\partial \varphi^{\beta}}{\partial \lambda} / \frac{\partial \varphi^{\beta}}{\partial x^{\alpha}} \epsilon_{\alpha \rho}$ and $\partial_{\rho} H_{\tau}=\frac{\partial \varphi^{\beta}}{\partial \tau} / \frac{\partial \varphi^{\beta}}{\partial x^{\alpha}} \epsilon_{\alpha \rho}$, where the Greek indices are 1 and 2 and where $\varphi$ 's are a two parameter family of symplectic diffeomorphisms given by the transformations, $x \rightarrow \varphi^{1}(x, y, \lambda, \tau)$ and $y \rightarrow \varphi^{2}(x, y, \lambda, \tau)$. Using these relations we write the action as

$$
\begin{gathered}
S=\frac{-1}{4 \pi^{2}} \int b(x, y)\left(\left(\frac{\partial \varphi^{\beta}}{\partial \lambda} / \frac{\partial \varphi^{\beta}}{\partial y}\right)\left(\frac{\partial \varphi^{\alpha}}{\partial \tau} / \frac{\partial \varphi^{\alpha}}{\partial x}\right)-\left(\frac{\partial \varphi^{\beta}}{\partial \tau} / \frac{\partial \varphi^{\beta}}{\partial y}\right)\left(\frac{\partial \varphi^{\alpha}}{\partial \lambda} / \frac{\partial \varphi^{\alpha}}{\partial x}\right)\right) d^{4} x \\
\frac{-1}{4 \pi^{2}} \int \operatorname{Tr} \gamma(x, y)\left(\left(\frac{\partial \varphi^{\beta}}{\partial \lambda} / \frac{\partial \varphi^{\beta}}{\partial x^{\alpha}}\right) \partial_{\alpha}\left(g^{-1} \partial_{\tau} g\right)-\left(\frac{\partial \varphi^{\beta}}{\partial \tau} / \frac{\partial \varphi^{\beta}}{\partial x^{\alpha}}\right) \partial_{\alpha}\left(g^{-1} \partial_{\lambda} g\right)\right. \\
\left.+\left[g^{-1} \partial_{\lambda} g, g^{-1} \partial_{\tau} g\right]\right) d^{4} x \\
\frac{-c\left(k, c_{v}, 26\right) \tilde{t}}{48 \pi^{2}} \int \frac{1}{y} \partial_{x}\left(\frac{\partial \varphi^{\alpha}}{\partial \lambda} / \frac{\partial \varphi^{\alpha}}{\partial x}\right) \partial_{x}^{2}\left(\frac{\partial \varphi^{\beta}}{\partial \tau} / \frac{\partial \varphi^{\beta}}{\partial x}\right) d^{4} x \\
\frac{-k \tilde{t}}{4 \pi^{2}} \int \frac{1}{y} \partial_{x}\left(g^{-1} \partial_{\lambda} g\right)\left(g^{-1} \partial_{\tau} g\right) d^{4} x .
\end{gathered}
$$

This is the result that we seek. 
We may use this action to study four dimensional gravity by considering an $\mathrm{SU}(2)$ Kac-Moody algebra. The spin connections will be $\mathrm{SU}(2)$ valued and again we may write Eq. (11) as a BF theory. To see this recall that $[2,3]$ the action corresponding to gravity via Ashekar variables may be written as

$$
\begin{aligned}
S & =\frac{1}{2} \int_{R^{4}} \operatorname{Tr} \Sigma_{\wedge} F \\
& =\frac{1}{2} \int d^{3} x d t \epsilon^{a b c}\left\{\operatorname{Tr}\left(\Sigma_{b c} F_{0 a}\right)+\operatorname{Tr}\left(\Sigma_{0 a} F_{b c}\right)\right\}
\end{aligned}
$$

where $\Sigma$ is an antisymmetric tensor field for the frame fields and $F$ is the curvature two form for real $\mathrm{SU}(2)$ valued spin connections. By identifying B of the BF theory with $\Sigma$ we may write

$$
\begin{aligned}
B_{0 a} & =\Sigma_{0 a}=\left(\frac{\partial \varphi^{\beta}}{\partial \lambda} / \frac{\partial \varphi^{\beta}}{\partial x}\right)\left(\frac{\partial \varphi^{\alpha}}{\partial \tau} / \frac{\partial \varphi^{\alpha}}{\partial y}\right)-\left(\frac{\partial \varphi^{\beta}}{\partial \tau} / \frac{\partial \varphi^{\beta}}{\partial x}\right)\left(\frac{\partial \varphi^{\alpha}}{\partial \lambda} / \frac{\partial \varphi^{\alpha}}{\partial y}\right) \\
B_{b c} & =\Sigma_{b c}=\gamma(x, y) \\
F_{0 a} & =\left(\left(\frac{\partial \varphi^{\beta}}{\partial \lambda} / \frac{\partial \varphi^{\beta}}{\partial x^{\alpha}}\right) \partial_{\alpha}\left(g^{-1} \partial_{\tau} g\right)-\left(\frac{\partial \varphi^{\beta}}{\partial \tau} / \frac{\partial \varphi^{\beta}}{\partial x^{\alpha}}\right) \partial_{\alpha}\left(g^{-1} \partial_{\lambda} g\right)+\left[g^{-1} \partial_{\lambda} g, g^{-1} \partial_{\tau} g\right]\right) \\
F_{b c} & =b(x, y)
\end{aligned}
$$

where we have suppressed explicit $\mathrm{SL}(2, \mathrm{R})$ indices and generators. With this observation, Eq. (11) can be used to study 4D gravity of the type described by Ashtekar and its order $\hbar$ contributions. From the evidence of the Atiyah-Singer index theorems, we suspect that one has integrated both spin one-half and three-half fields coupled to the connection. This is because the spin $\frac{1}{2}$ sector alone admits no anomaly while the $\frac{3}{2}$ sector does. This does not necessarily imply that one needs supergravity, but that the Rarita-Schwinger field is on the same fundamental footing as the Dirac (Weyl) fermions. Let us also emphasize that the gauge group in Eq. (11) is arbitrary so that we may study any self-dual gauge theory.

Notice that the last two terms of Eq. (11) are exactly analogous to the Polyakov and WZNW terms. The Polyakov term is just two separate 2D type terms plus a cross term between $\varphi^{1}$ and $\varphi^{2}$ contributions [9]. Since the y-integration only serves to pick up the $W_{2}$ sector, we may think of these Polyakov terms as a generalization of the interacting $2 \mathrm{D}$ gravities. A generalization to this has been computed in [10] for the $w_{\infty}$ algebra 
that includes central extensions for all conformal fields. We prefer to leave the action in a form that is quadratic in the frame fields. The very last term is just the WZNW model when one expands all terms and integrates by parts. The y-integration demands that only the $W^{2}$ sector or, if you wish, the quadratic differentials corresponding to the space of metrics, pick up the anomaly.

Other uses of this action would be to identify the gravitational instantons and monopoles by using the type of analysis that we used for QCD strings. By demanding that $\Sigma$ and $\mathrm{F}$ be proportional to each other, we can identify the isotropy group of the coadjoint vector and extract gravitational instantons. The isotropy equation, Eq. (8), may dictate inequivalent vacua for $4 \mathrm{D}$ gravity. Different isotropy groups correspond to different semi-classical vacua. Furthermore different topologies can be studied by changing the representation of SDiff $R^{2}$ to other structures like SDiff $T^{2}$. We will focus on these differences and the relationship to gravitational instantons in a future publication.

We have seen that the method of coadjoint orbits can lead us to order $\hbar$ effects of a field theory with a rich symmetry. The method seems to indicate that the path integral can be computed (up to constants) rather painlessly to arrive at effective actions. These effective actions may be seen as a bosonization prescription (since the fermionic degrees of freedom are replaced by bosonic degrees of freedom), but perhaps they also contain contributions from the bound states of the fermions. Indeed the QCD discussion leads one to think that the string theory is a string theory of mesons. The analog of this statement for the $4 \mathrm{D}$ gravity sector would imply that the spin connections and frame fields are bound states of more primitive fermionic fields. The covectors may serve as external fields and we may promote them to dynamical fields by adding an appropriate kinetic term. Another point of interest is that we were able to show that the WZNW models and 2D quantum gravity are quantum corrections to some ChernSimons theories in 3D. A statement similar to this appears at the $4 \mathrm{D}$ level for the BF theories. In the spirit of using algebraic techniques to understand functional integrals we will use the Moyal brackets [22] to get all higher order corrections to these theories [23]. This is in accord with the spirit of Ref. [24]. This idea was used in the frame work of coadjoint orbits to extend $w_{\infty}$ to $W_{\infty}$ in Ref. [10]. There one finds that (correcting 
errors in Ref. [10], for $H_{\lambda}$ and $H_{\tau}$ (see Eq. 10)), the $W_{\infty}$ effective action for the orbit corresponding to the coadjoint vector $\mathbf{B}=\left(\tilde{B}, t^{l} \tilde{\alpha}_{l}\right)$ is

$$
\begin{aligned}
& S(\tilde{B})= \\
& \sum_{i=0}^{\infty} t^{i} \frac{c_{i}}{4 \pi^{2}} \oint \oint \iint D_{y}^{2(i+1)} \partial_{x}^{i+1}\left(y^{i+1} H_{\tau}\right) D_{y}^{2 i+2} \partial_{x}^{i+2}\left(y^{i+2} H_{\lambda}\right) d x d y d \lambda d \tau \\
& +\oint \oint \iint \tilde{B}(x, y)\left\{H_{\tau}, H_{\lambda}\right\}_{\hbar} d x d y d \lambda d \tau,
\end{aligned}
$$

where the derivative operator $D_{y}=\partial_{y}+\frac{x}{y} \partial_{x}$.

Lastly, these generalizations may further provide insight into how one extends the theorems of Ref. [14] to non-classical groups. In particular, the 4D BF action suggests a relationship between maps from $C P^{1} \rightarrow$ Diff $S^{1}$ and self-dual Einstein theories, where the $2 \mathrm{D}$ action corresponds to two separate Liouville-Polyakov theories and an interacting term between them that appears as a WZ type term. Also the SU(2) case of Eq. (11) is suitable for studying a field theoretic version of reducible, self-dual connections on compact four manifolds by choosing the isotropy group of the $\mathbf{B}$ field to be $\mathbf{U}(1)$.

Acknowledgements V.G.J.R would like to thank A.P. Balachandran, S.J. Gates, D. Karabali, V.P. Nair, P. Srinivasan for discussion and encouragement. This work was supported in part by NSF Grant PHY-9103914. 


\section{REFERENCES}

[1] E. Witten, Nucl. Phys. B311 46 (1988/89)

[2] J. Samuel, Pramãna - J. Phys. 28 L429 (1987)

[3] Ted Jacobson and Lee Smolin, Class. Quant. Grav. 5583 (1988)

[4] A.P. Balachandran and P. Teotonio Sobrinho,

The Edge States of the BF System and the London Equations

Syracuse Preprint SU-4228-491

[5] F. Zaccoria, E.C.G. Sudarshan, J.S. Nilsson, N. Mukunda, G. Marmo, and A.P. Balachandran, Phys. Rev D27 2327 (1983);

[6] A.P. Balachandran, G. Marmo, B.S. Skagerstam, and A. Stern,

Gauge Symmetries and Fibre Bundles; Applications to Particle Dynamics, Springer-Verlag Berlin (1983)

[7] B. Rai and V.G.J. Rodgers, Nucl. Phys. B341 119 (1990);

A. Yu Alekseev and S.L. Shatashvili, Nucl. Phys. B323 719 (1989)

[8] G.W. Delius, P. van Nieuwenhuizen, and V.G.J. Rodgers,

Inter. Jour. of Mod. Physics A5 3943 (1990)

[9 ] V.G.J Rodgers, Mod. Phys. Lett. A 61045 (1991)

[10] V.G.J. Rodgers, Mod. Phys. Lett. A 61893 (1991)

[11] D. Gepner and E. Witten, Nucl. Phys. B 278493 (1986)

[12] D. Birmingham, M. Blau, M. Rakowski, and G. Thompson, Phys. Rep. 209129 (1991)

[13] V.G.J. Rodgers, QCD Instantons and 2D Surfaces,

Iowa Preprint UI92-2, to appear in Mod. Phys. Lett. A

[14] M. Atiyah, Comm. Math. Phys. 93 (1984) 437

[15] P. Goddard and D. Olive, Int. J. Mod. Phys. A 1303 (1986)

[16] Q.H. Park, Phys. Lett. 236 B 429 (1990); Phys. Lett. 238 B 287 (1990);

Phys. Lett. 257 B 105 (1991)

[17] K. Yamagishi and G.F. Chapline, Class. Quantum Grav. 8427 (1991)

[18] K. Yamagishi, Phys. Lett. B 259436 (1991)

[19] H. Ooguri and C. Vafa, Mod. Phys. Lett. A5 1389 (1990);

Nucl. Phys. B361 469 (1991); Nucl. Phys. B367 83 (1991)

[20] E. Sezgin, Area-Preserving Diffeomorphisms, $w_{\infty}$ Algebras and $w_{\infty}$ Gravity

Trieste Summer School Lecture, July 17 - Aug. 9, 1991

[21] Abhay Ashtekar, "Non-Perturbative Canonical Gravity," World Scientific (1991)

[22] J. Moyal, Proc. Comb. Phil. Soc. 4599 (1949)

I. Bakas, Comm. Math. Phys. 134487 (1990), Phys. Lett. B228 57 (1989)

[23] R.P. Lano and V.G.J. Rodgers, in progress

[24] E. Bergshoeff, E. Sezgin, Y. Tanii and P.K. Townsend, Ann. Phys. 199340 (1990) 